\title{
Multiple periodic solutions for second-order discrete Hamiltonian systems
}

\author{
Da-Bin Wang*, Man Guo \\ Department of Applied Mathematics, Lanzhou University of Technology, 730050 Lanzhou, People's Republic of China.
}

Communicated by D. Baleanu

\begin{abstract}
By applying critical point theory, the multiplicity of periodic solutions to second-order discrete Hamiltonian systems with partially periodic potentials was considered. It is noticed that, in this paper, the nonlinear term is growing linearly and main results extend some present results. (C)2017 all rights reserved.
\end{abstract}

Keywords: Discrete Hamiltonian systems, periodic solutions, the generalized saddle point theorem. 2010 MSC: 34C25, 58E50.

\section{Introduction}

Consider the following systems

$$
\Delta^{2} x(t-1)+\nabla G(t, x(t))=0, \quad t \in \mathbb{Z},
$$

where $\Delta x(t)=x(t+1)-x(t), \Delta^{2} x(t)=\Delta(\Delta x(t))$. For any $t \in \mathbb{Z}, G: \mathbb{Z} \times \mathbb{R}^{N} \rightarrow \mathbb{R}$ is $C^{1}$ in $x$, and $\mathrm{G}(\mathrm{t}+\mathrm{T}, \mathrm{x})=\mathrm{G}(\mathrm{t}, \mathrm{x})$ for any $x \in \mathbb{R}^{\mathrm{N}}$, where $\mathrm{T} \in \mathbb{Z}$ and $\mathrm{T}>0$.

As far as we known, Guo and $\mathrm{Yu}$ [6] obtained the first variational result about T-periodic solutions for system (1.1). Soon afterwards, applying variational methods, there have been many studies in the literature consider about periodic solutions to discrete systems [2-6, 9, 11, 14-18].

It is noticed that the existence of one periodic solution to system (1.1) were obtained in [6] in case $\nabla \mathrm{G}(\mathrm{t}, \mathrm{x})$ is bounded. Afterward, in [14, 15], Xue and Tang studied the system (1.1), in which $\nabla \mathrm{G}$ is growing sublinearly: there exist $\delta>0, \eta>0$ satisfying

$$
|\nabla G(t, u)| \leqslant \delta|u|^{\alpha}+\eta, \quad \forall(t, u) \in[1, T] \cap \mathbb{Z} \times \mathbb{R}^{N},
$$

where $\alpha \in[0,1)$. Furthermore, $G$ satisfies:

$$
\lim _{|u| \rightarrow \infty}|u|^{-2 \alpha} \sum_{t=1}^{T} G(t, u)=+\infty,
$$

\footnotetext{
${ }^{*}$ Corresponding author

Email addresses: wangdb96@163.com (Da-Bin Wang), guoman615@163.com (Man Guo)
} 
or

$$
\lim _{|u| \rightarrow \infty}|u|^{-2 \alpha} \sum_{t=1}^{T} G(t, u)=-\infty
$$

In case $\nabla G$ is growing sublinearly or linearly (that is $\nabla G$ satisfies (1.2) with $\alpha=1$ ), Tang and Zhang [11] extended the main results obtained in $[6,14,15]$ under more weakened conditions on G:

$$
\lim _{|u| \rightarrow \infty}|u|^{-2 \alpha} \sum_{t=1}^{T} G(t, u)<+\infty
$$

or

$$
\lim _{|u| \rightarrow \infty}|u|^{-2 \alpha} \sum_{t=1}^{T} G(t, u)>-\infty
$$

Recently, in [16], under $\nabla \mathrm{G}(\mathrm{t}, \mathrm{u})$ satisfies (1.2), $\mathrm{G}$ is coercive or resonant and periodic only in a part of the variables, that is, there exists an integer $k \in[0, N]$ such that:

(i) $G(t, u)$ is $T_{j}$-periodic in $u_{j}, 1 \leqslant j \leqslant k$.

$$
|\mathrm{u}|^{-2 \alpha} \sum_{\mathrm{t}=1}^{\mathrm{T}} \mathrm{G}(\mathrm{t}, \mathrm{u}) \rightarrow \pm \infty \text { as }|\mathrm{u}| \rightarrow \infty, \mathrm{u} \in\{0\} \times \mathbb{R}^{\mathrm{N}-\mathrm{k}},
$$

by using generalized saddle point theorem [8], Yan et al. considered the multiple periodic solutions for system (1.1) and got some interesting results.

Motivated by [6,11,14-16], especially by [11, 16], one natural question is: What will happen when $\nabla G$ is growing linearly and $G$ is coercive or resonant and periodic only in a part of the variables? More concretely, can we obtain some results similar to that of in [16] with $\nabla \mathrm{G}$ is growing linearly? It seems one interesting question. In this paper, we will state them.

Theorem 1.1. Suppose that there exists an integer $k \in[0, \mathrm{~N}]$ such that

$\left(\mathrm{H}_{1}\right) \mathrm{G}(\mathrm{t}, \mathrm{u})$ is $\mathrm{T}_{\mathrm{j}}$-periodic in $\mathrm{u}_{\mathrm{j}}, 1 \leqslant \mathrm{j} \leqslant \mathrm{k}$.

$\left(\mathrm{H}_{2}\right)$ There exist constants $0<\delta<\frac{\lambda_{1}}{4}, \eta>0$ satisfying

$$
|\nabla \mathrm{G}(\mathrm{t}, \mathrm{u})| \leqslant \delta|\mathrm{u}|+\eta, \forall(\mathrm{t}, \mathrm{u}) \in[1, \mathrm{~T}] \cap \mathbb{Z} \times \mathbb{R}^{\mathrm{N}}
$$

here $\lambda_{l}=2-2 \cos l \omega, \omega=\frac{2}{T}, l \in[0,[T / 2]] \cap \mathbb{Z},[\cdot]$ is the integral function.

$$
\liminf _{|u| \rightarrow \infty}|u|^{-2} \sum_{t=1}^{T} G(t, u)>\frac{\lambda_{[T / 2]} T}{4}+\frac{\delta^{2} T}{\lambda_{[T / 2]}}+\delta T, u \in\{0\} \times \mathbb{R}^{N-k} .
$$

Then the Hamiltonian system (1.1) possesses $k+1$ periodic solutions.

Theorem 1.2. Suppose that $\mathrm{G}$ satisfies $\left(\mathrm{H}_{1}\right),\left(\mathrm{H}_{2}\right)$, and

$\left(\mathrm{H}_{4}\right)$

$$
\limsup _{|u| \rightarrow \infty}|u|^{-2} \sum_{t=1}^{T} G(t, u)<-\frac{\delta T}{2}, u \in\{0\} \times \mathbb{R}^{N-k} .
$$

Then the Hamiltonian system (1.1) possesses $k+1$ periodic solutions.

\section{Definitions and lemmas}

Let

$$
\mathrm{H}_{\mathrm{T}}=\left\{x: \mathbb{Z} \rightarrow \mathbb{R}^{\mathrm{N}} \mid x(\mathrm{t}+\mathrm{T})=x(\mathrm{t}), \quad \forall \mathrm{t} \in \mathbb{Z}\right\},
$$


and

$$
\langle x, y\rangle=\sum_{t=1}^{\mathrm{T}}(x(t), y(t)),\|x\|=\left(\sum_{t=1}^{\mathrm{T}}|x(t)|^{2}\right)^{1 / 2}, \forall x, y \in \mathrm{H}_{\mathrm{T}} .
$$

Obviously, $\left(\mathrm{H}_{\mathrm{T}},\langle\cdot, \cdot\rangle\right)$ is a Hilbert space (in fact, is finite-dimensional space).

For $x \in \mathrm{H}_{\mathrm{T}}$, denote

$$
x_{1}=\frac{1}{T} \sum_{t=1}^{T}|x(t)|, \quad x_{2}(t)=x(t)-x_{1} .
$$

Let $\left\{e_{j} \mid 1 \leqslant j \leqslant N\right\}$ be the canonical basis of $\mathbb{R}^{N}$, and $k_{j}$ be the unique integer such that

$$
0 \leqslant\left(x_{1}, e_{j}\right)-k_{j} T_{j}<T_{j} \text { for } 1 \leqslant j \leqslant k .
$$

Set

$$
\tilde{x}(t)=P x_{1}+Q x_{1}+x_{2}(t)
$$

for all $x \in H_{T}$, where

$$
P x_{1}=\sum_{j=k+1}^{N}\left(x_{1}, e_{j}\right) e_{j}, \quad Q x_{1}=\sum_{j=1}^{k}\left(\left(x_{1}, e_{j}\right)-k_{j} T_{j}\right) e_{j} .
$$

It is easy to see that there exists $\mu>0$ such that

$$
\left|\mathrm{Q} x_{1}\right|<\mu .
$$

Let $\mathrm{I}$ be defined on $\mathrm{H}_{\mathrm{T}}$ by

$$
I(x)=-\frac{1}{2} \sum_{t=1}^{T}|\Delta x(t)|^{2}+\sum_{t=1}^{T} G(t, x(t)) .
$$

Then

$$
\left\langle\mathrm{I}^{\prime}(\mathrm{x}), \mathrm{y}\right\rangle=-\sum_{\mathrm{t}=1}^{\mathrm{T}}(\Delta x(\mathrm{t}), \Delta \mathrm{y}(\mathrm{t}))+\sum_{\mathrm{t}=1}^{\mathrm{T}}(\nabla \mathrm{G}(\mathrm{t}, \mathrm{x}(\mathrm{t})), \mathrm{y}(\mathrm{t})),
$$

for any $x, y \in H_{T}$. According to fact that of in [15], the periodic solutions for system (1.1) are critical points for the functional I.

Denote

$$
F=\left\{\sum_{j=1}^{k} l_{j} T_{j} e_{j} \mid l_{j} \in \mathbb{Z}, 1 \leqslant j \leqslant k\right\} .
$$

Let $\pi: \mathrm{H}_{\mathrm{T}} \rightarrow \mathrm{H}_{\mathrm{T}} / \mathrm{F}$ be the canonical surjection (in fact, $\mathrm{F}$ is a discrete subgroup of $\mathrm{H}_{\mathrm{T}}$ ).

In fact, $\mathrm{H}_{\mathrm{T}} / \mathrm{F}=\mathrm{U} \times \mathrm{V}$, here

$$
\begin{aligned}
\mathrm{U} & =\mathrm{E}+\mathrm{W}, \\
\mathrm{W} & =\mathrm{H}_{\mathrm{T}}^{1}=\left\{x \in \mathrm{H}_{\mathrm{T}} \mid x_{1}=0\right\}, \\
\mathrm{E} & =\operatorname{span}\left\{e_{\mathrm{k}+1}, \ldots, e_{\mathrm{N}}\right\},
\end{aligned}
$$

and

$$
\mathrm{V}=\operatorname{span}\left\{e_{1}, \ldots, e_{\mathrm{k}}\right\} / \mathrm{F} .
$$

It is easy to see that $V$ and the torus $T^{k}$ are isomorphic. Let $f(\pi(x))=I(x)$, in fact $f: U \times V \rightarrow \mathbb{R}$.

Lemma 2.1 (The generalized saddle point theorem [8]). Let $\mathrm{U}$ be a Banach space with a decomposition $\mathrm{U}=$ $\mathrm{E}+\mathrm{W}$, where $\mathrm{E}$ and $\mathrm{W}$ are two subspaces of $\mathrm{U}$ with $\operatorname{dim} \mathrm{W}<+\infty$. Let $\mathrm{V}$ be a finite-dimensional, and compact 
$\mathrm{C}^{2}$-manifold without boundary. Let $\mathrm{f}: \mathrm{U} \times \mathrm{V} \rightarrow \mathbb{R}$ be a $\mathrm{C}^{1}$-function and satisfy the (P.S.) condition. If there exist $\rho>0, \gamma<\beta$ satisfy

(a) $\inf _{x \in E \times V} f(x) \geqslant \beta ;$

(b) $\sup _{x \in S \times V} f(x) \leqslant \gamma$,

where $S=\partial D, D=\{z \in W|| z \mid \leqslant \rho\}$. Then the functional $\varphi$ has at least cuplength $(V)+1$ critical points.

Lemma 2.2 (Theorem 4.12 in [10]). Let $\varphi \in \mathrm{C}^{1}\left(\mathrm{H}_{\mathrm{T}}, \mathbb{R}\right)$ be a $\mathrm{G}$-invariant functional $(\varphi(\mathrm{u}+\mathrm{g})=\varphi(\mathrm{u})$ for every $\mathrm{u} \in \mathrm{H}_{\mathrm{T}}$ and $\mathrm{g} \in \mathrm{F}$ ) satisfying the (P.S. $)_{\mathrm{G}}$ condition (that is every sequence $\left\{x_{\mathrm{n}}\right\}$ of $\mathrm{H}_{\mathrm{T}}$ such that $\varphi\left(\mathrm{x}_{\mathrm{n}}\right)$ is bounded, $\varphi^{\prime}\left(x_{n}\right) \rightarrow 0$ as $n \rightarrow \infty, \pi\left(x_{n}\right)$ has a convergent subsequence). If $\varphi$ is bounded from below and if the dimension $k$ of the space generated by $\mathrm{F}$ is finite, then $\varphi$ has at least $\mathrm{k}+1$ critical orbits.

Lemma 2.3. Let

$$
M_{r}:=\left\{x \in H_{\mathrm{T}}:-\Delta^{2} x(t-1)=\lambda_{r} x(t)\right\},
$$

here $\lambda_{\mathrm{r}}$ are defined as in condition $\left(\mathrm{H}_{2}\right)$. Then $\mathrm{M}_{\mathrm{r}}$ is a subspace of $\mathrm{H}_{\mathrm{T}}$ and

(i) $M_{r} \perp M_{i}, r \neq i, r, i \in[0,[T / 2]] \cap \mathbb{Z}$;

(ii) $\mathrm{H}_{\mathrm{T}}=\oplus_{\mathrm{r}=0}^{[\mathrm{T} / 2]} \mathrm{M}_{\mathrm{r}}$.

Lemma 2.4 ([15]). Let

$$
\mathrm{H}_{\mathrm{r}}=\oplus_{\mathrm{i}=0}^{\mathrm{r}} M_{i}, \quad \mathrm{H}_{\mathrm{r}}^{\perp}=\oplus_{i=\mathrm{r}+1}^{[\mathrm{T} / 2]} M_{i},
$$

where $r \in[0,[\mathrm{~T} / 2]] \cap \mathbb{Z}$. Then

$$
\sum_{t=1}^{\mathrm{T}}|\Delta x(\mathrm{t})|^{2} \leqslant \lambda_{\mathrm{r}}\|x\|^{2}, \quad \forall x \in \mathrm{H}_{\mathrm{r}}
$$

and

$$
\sum_{t=1}^{\mathrm{T}}|\Delta x(\mathrm{t})|^{2} \geqslant \lambda_{\mathrm{r}+1}\|x\|^{2}, \quad \forall x \in \mathrm{H}_{\mathrm{r}}^{\perp}
$$

\section{Proof of theorems}

Proof of Theorem 1.1.

Step 1. We will assert the (P.S.) condition holds.

Assume that $\left\{\pi\left(x_{n}\right)\right\}$ is a (P.S.) sequence of $f$, i.e., $I\left(x_{n}\right)$ is bounded, $I^{\prime}\left(x_{n}\right) \rightarrow 0$. Combing $\left(H_{3}\right)$ with $\lambda_{1}>4 \delta$, we have that

$$
\liminf _{|\mathfrak{u}| \rightarrow \infty}|u|^{-2} \sum_{t=1}^{T} G(t, u)>\frac{4 \delta^{2} T \lambda_{[T / 2]}}{\lambda_{1}^{2}}+\frac{\delta^{2} T}{\lambda_{[T / 2]}}+\frac{16 \delta^{3} T}{\lambda_{1}^{2}} .
$$

By $\left(\mathrm{H}_{2}\right)$ and (3.1), one has that

$$
\begin{aligned}
\left|\sum_{t=1}^{T}\left(G(t, \tilde{x}(t))-G\left(t, P x_{1}\right)\right)\right| & \leqslant \sum_{t=1}^{T}\left|G(t, \tilde{x}(t))-G\left(t, P x_{1}\right)\right| \\
& \leqslant \sum_{t=1}^{T}\left|\int_{0}^{1}\left(\nabla G\left(t, P x_{1}+s\left(Q x_{1}+x_{2}(t)\right)\right), Q x_{1}+x_{2}(t)\right) d s\right| \\
& \leqslant \sum_{t=1}^{T} \int_{0}^{1}\left|\left(\nabla G\left(t, P x_{1}+s\left(Q x_{1}+x_{2}(t)\right)\right), Q x_{1}+x_{2}(t)\right)\right| d s
\end{aligned}
$$




$$
\begin{aligned}
\leqslant & \sum_{t=1}^{T} \int_{0}^{1}\left|\nabla G\left(t, P x_{1}+s\left(Q x_{1}+x_{2}(t)\right)\right) \| Q x_{1}+x_{2}(t)\right| d s \\
\leqslant & \sum_{t=1}^{T} \int_{0}^{1}\left(\delta\left|P x_{1}+s\left(Q x_{1}+x_{2}(t)\right)\right|+\eta\right)\left|Q x_{1}+x_{2}(t)\right| d s \\
\leqslant & \sum_{t=1}^{T} \delta\left(\left|P x_{1}\right|+\left|Q x_{1}+x_{2}(t)\right|\right)\left|Q x_{1}+x_{2}(t)\right|+\sum_{t=1}^{T} \eta\left|Q x_{1}+x_{2}(t)\right| \\
\leqslant & \delta \mu T\left|P x_{1}\right|+\sum_{t=1}^{T} \delta\left|P x_{1} \| x_{2}(t)\right|+\sum_{t=1}^{T} 4 \delta\left(\left|Q x_{1}\right|^{2}+\left|x_{2}(t)\right|^{2}\right) \\
& +\sum_{t=1}^{T} \eta\left(\left|Q x_{1}\right|+\left|x_{2}(t)\right|\right) \\
\leqslant & \delta \mu T\left|P x_{1}\right|+\sum_{t=1}^{T} \delta\left|P x_{1} \| x_{2}(t)\right|+4 \delta \mu^{2} T+4 \delta \sum_{t=1}^{T}\left|x_{2}(t)\right|^{2} \\
& +\eta \mu T+\sum_{t=1}^{T} \eta\left|x_{2}(t)\right| \\
\leqslant & \delta \mu T\left|P x_{1}\right|+\frac{\delta^{2} T}{\lambda_{[T / 2]}}\left|P x_{1}\right|^{2}+\frac{\lambda_{[T / 2]}}{2}\left\|x_{2}\right\|^{2}+4 \delta \mu^{2} T+4 \delta\left\|x_{2}\right\|^{2} \\
& +\eta \mu T+\eta \sqrt{T}\left\|x_{2}\right\| .
\end{aligned}
$$

In the same way, we get

$$
\begin{aligned}
\left|\sum_{t=1}^{T}\left(\nabla G(t, \tilde{x}(t)), x_{2}(t)\right)\right| & =\left|\sum_{t=1}^{T}\left(\nabla G\left(t, P x_{1}+Q x_{1}+x_{2}(t)\right), x_{2}(t)\right)\right| \\
& \leqslant \sum_{t=1}^{T}\left|\nabla G\left(t, P x_{1}+Q x_{1}+x_{2}(t)\right) \| x_{2}(t)\right| \\
& \leqslant \sum_{t=1}^{T}\left(\delta\left|P x_{1}+Q x_{1}+x_{2}(t)\right|+\eta\right)\left|x_{2}(t)\right| \\
& \leqslant \sum_{t=1}^{T} \delta\left|P x_{1}\left\|\left.x_{2}(t)\left|+\delta \mu \sqrt{T}\left\|x_{2}\right\|+\delta \sum_{t=1}^{T}\right| x_{2}(t)\right|^{2}+\eta \sqrt{T}\right\| x_{2} \|\right. \\
& \leqslant \frac{\lambda_{1}}{2}\left\|x_{2}\right\|^{2}+\frac{\delta^{2} T}{\lambda_{1}}\left|P x_{1}\right|^{2}+\delta\left\|x_{2}\right\|^{2}+(\delta \mu+\eta) \sqrt{T}\left\|x_{2}\right\| .
\end{aligned}
$$

Since the fact $x_{1 n} \in \mathrm{H}_{0}$ and $x_{2 n} \in \mathrm{H}_{0}^{\perp}$, by (2.3), one has that

$$
\sum_{t=1}^{T}\left(\Delta x_{n}(t), \Delta x_{2 n}(t)\right)=\sum_{t=1}^{T}\left(\Delta x_{2 n}(t), \Delta x_{2 n}(t)\right)=\sum_{t=1}^{T}\left|\Delta x_{2 n}(t)\right|^{2} \geqslant \lambda_{1}\left\|x_{2 n}\right\|^{2} .
$$

By $\mathrm{I}\left(\mathrm{x}_{\mathrm{n}}\right)$ is bounded, $\mathrm{I}^{\prime}\left(\mathrm{x}_{\mathrm{n}}\right) \rightarrow 0$ and (3.3), for $\mathrm{n}$ large enough, one gets

$$
\begin{aligned}
\sum_{t=1}^{T}\left(\Delta x_{n}(t), \Delta x_{2 n}(t)\right) & =-\left\langle I^{\prime}\left(x_{n}\right), x_{2 n}\right\rangle+\sum_{t=1}^{T}\left(\nabla G\left(t, x_{n}(t)\right), x_{2 n}(t)\right) \\
& =-\left\langle I^{\prime}\left(x_{n}\right), x_{2 n}\right\rangle+\sum_{t=1}^{T}\left(\nabla G\left(t, \tilde{x}_{n}(t)\right), x_{2 n}(t)\right) \\
& \leqslant\left\|x_{2 n}\right\|+\frac{\lambda_{1}}{2}\left\|x_{2 n}\right\|^{2}+\frac{\delta^{2} T}{\lambda_{1}}\left|P x_{1 n}\right|^{2}+\delta\left\|x_{2 n}\right\|^{2}+(\delta \mu+\eta) \sqrt{T}\left\|x_{2 n}\right\| .
\end{aligned}
$$


Combing (3.4) with (3.5), one has that

$$
\left(\frac{\lambda_{1}}{2}-\delta\right)\left\|x_{2 n}\right\|^{2}-((\delta \mu+\eta) \sqrt{T}+1)\left\|x_{2 n}\right\| \leqslant \frac{\delta^{2} T}{\lambda_{1}}\left|P x_{1 n}\right|^{2} .
$$

Therefore, there exists $C_{1}$ such that

$$
\frac{\delta^{2} \mathrm{~T}}{\lambda_{1}}\left|\mathrm{P} x_{1 n}\right|^{2} \geqslant \frac{\lambda_{1}}{4}\left\|\mathrm{x}_{2 \mathrm{n}}\right\|^{2}+\mathrm{C}_{1}
$$

for all large $n$, where

$$
C_{1}=\min _{s \in[0,+\infty)}\left\{\left(\frac{\lambda_{1}}{4}-\delta\right) s^{2}-((\delta \mu+\eta) \sqrt{\top}+1) s\right\} .
$$

Since $\lambda_{1}>4 \delta,-\infty<C_{1}<0$, it follows from (3.6) that

$$
\left\|x_{2 n}\right\|^{2} \leqslant \frac{4 \delta^{2} T}{\lambda_{1}^{2}}\left|P x_{1 n}\right|^{2}-\frac{4 C_{1}}{\lambda_{1}} .
$$

Then

$$
\left\|x_{2 n}\right\| \leqslant \frac{2 \delta \sqrt{T}}{\lambda_{1}}\left|P x_{1 n}\right|+C_{2}
$$

where $0<\mathrm{C}_{2}<+\infty$.

So by the boundedness of $I\left(x_{n}\right),(2.2),(3.2),(3.7)$, and (3.8), we have that

$$
\begin{aligned}
& C_{3} \geqslant I\left(x_{n}\right)=I\left(\tilde{x}_{n}\right)=-\frac{1}{2} \sum_{t=1}^{T}\left|\Delta \tilde{x}_{n}(t)\right|^{2}+\sum_{t=1}^{T} G\left(t, \tilde{x}_{n}(t)\right) \\
& =-\frac{1}{2} \sum_{t=1}^{T}\left|\Delta \tilde{x}_{n}(t)\right|^{2}+\sum_{t=1}^{T}\left[G\left(t, \tilde{x}_{n}(t)\right)-G\left(t, P x_{1 n}\right)\right]+\sum_{t=1}^{T} G\left(t, P x_{1 n}\right) \\
& \geqslant-\frac{1}{2} \lambda_{[T / 2]}\left\|x_{2 n}\right\|^{2}-\delta \mu T\left|P x_{1 n}\right|-\frac{\delta^{2} T}{\lambda_{[T / 2]}}\left|\mathrm{P} x_{1 n}\right|^{2}-\frac{\lambda_{[T / 2]}}{2}\left\|x_{2 n}\right\|^{2} \\
& -4 \delta \mu^{2} \mathrm{~T}-4 \delta\left\|x_{2 n}\right\|^{2}-\eta \mu \mathrm{T}-\eta \sqrt{\mathrm{T}}\left\|\mathrm{x}_{2 \mathrm{n}}\right\|+\sum_{\mathrm{t}=1}^{\mathrm{T}} \mathrm{F}\left(\mathrm{t}, \mathrm{P} \mathrm{x}_{1 \mathrm{n}}\right) \\
& \geqslant-\lambda_{[\mathrm{T} / 2]}\left(\frac{4 \delta^{2} \mathrm{~T}}{\lambda_{1}^{2}}\left|\mathrm{P} x_{1 \mathrm{n}}\right|^{2}-\frac{4 \mathrm{C}_{1}}{\lambda_{1}}\right)-\frac{\delta^{2} \mathrm{~T}}{\lambda_{[\mathrm{T} / 2]}}\left|\mathrm{P} x_{1 \mathrm{n}}\right|^{2}-\delta \mu \mathrm{T}\left|\mathrm{P} x_{1 \mathrm{n}}\right|-4 \delta \mu^{2} \mathrm{~T}-\eta \mu \mathrm{T} \\
& -4 \delta\left(\frac{4 \delta^{2} \mathrm{~T}}{\lambda_{1}^{2}}\left|\mathrm{P} x_{1 \mathrm{n}}\right|^{2}-\frac{4 \mathrm{C}_{1}}{\lambda_{1}}\right)-\eta \sqrt{\mathrm{T}}\left(\frac{2 \delta \sqrt{\mathrm{T}}}{\lambda_{1}}\left|\mathrm{P} x_{1 \mathrm{n}}\right|+\mathrm{C}_{2}\right)+\sum_{\mathrm{t}=1}^{\mathrm{T}} \mathrm{F}\left(\mathrm{t}, \mathrm{P} x_{1 \mathrm{n}}\right) \\
& \geqslant\left|\mathrm{P} x_{1 \mathrm{n}}\right|^{2}\left(\left|\mathrm{P} x_{1 \mathrm{n}}\right|^{-2} \sum_{\mathrm{t}=1}^{\mathrm{T}} \mathrm{F}\left(\mathrm{t}, \mathrm{P} x_{1 \mathrm{n}}\right)-\frac{4 \delta^{2} \mathrm{~T} \lambda_{[\mathrm{T} / 2]}}{\lambda_{1}^{2}}-\frac{\delta^{2} \mathrm{~T}}{\lambda_{[\mathrm{T} / 2]}}-\frac{16 \delta^{3} \mathrm{~T}}{\lambda_{1}^{2}}\right) \\
& -\mathrm{C}_{4}\left|\mathrm{P} x_{1 \mathrm{n}}\right|-\mathrm{C}_{5} \text {, }
\end{aligned}
$$

for $\mathrm{n}$ sufficient large, where $\mathrm{C}_{4}>0, \mathrm{C}_{5}>0$ are constants.

It follows from (3.9) and (3.1) that $\left|\mathrm{P} x_{1 n}\right|$ is bounded. Then by (3.8), we obtain $\left\|x_{2 n}\right\|$ is bounded, so $\left\{\tilde{x}_{n}\right\}$ is also bounded. Observe that $\pi\left(x_{n}\right)=\pi\left(\tilde{x}_{n}\right)$ and $H_{T}$ is a finite-dimensional space, then we obtain the result that $f$ satisfies the (P.S.) condition.

Step 2. To prove that (a) and (b) of Lemma 2.1 are satisfied. 
Since $\pi(x) \in E \times V, x=P x_{1}+Q x_{1}$, from $\left(H_{3}\right)$, we get

$$
f(\pi(x))=\sum_{t=1}^{T} G\left(t, P x_{1}+Q x_{1}\right) \rightarrow \infty
$$

uniformly for $\pi\left(Q x_{1}\right) \in V$ as $\left|P x_{1}\right| \rightarrow \infty$. So, there exists a constant $\beta$ satisfying $\inf _{\pi(x) \in E \times V} f(\pi(x)) \geqslant \beta$.

By $\left(\mathrm{H}_{2}\right), \exists \mathrm{C}_{6}>0$ satisfying

$$
|G(t, u)| \leqslant\left|\int_{0}^{1}(\nabla G(t, s u), u) d s\right|+G(t, 0) \leqslant \int_{0}^{1}|\nabla G(t, s u)||u| d s+G(t, 0) \leqslant \frac{\delta}{2}|u|^{2}+\eta|u|+C_{6},
$$

for all $t \in[1, T] \cap \mathbb{Z}, u \in \mathbb{R}^{N}$.

Since $\pi(x) \in W \times V, x=Q x_{1}+x_{2}$. Hence, by (2.3), (2.1), and (3.10), one has that

$$
\begin{aligned}
f(\pi(x))=I(x)=I\left(Q x_{1}+x_{2}\right) & =-\frac{1}{2} \sum_{t=1}^{T}\left|\Delta x_{2}(t)\right|^{2}+\sum_{t=1}^{T} G\left(t, Q x_{1}+x_{2}(t)\right) \\
& \leqslant-\frac{1}{2} \lambda_{1}\left\|x_{2}\right\|^{2}+\sum_{t=1}^{T} \frac{\delta}{2}\left|Q x_{1}+x_{2}(t)\right|^{2}+\sum_{t=1}^{T} \eta\left|Q x_{1}+x_{2}(t)\right|+C_{6} T \\
& \leqslant-\frac{1}{2} \lambda_{1}\left\|x_{2}\right\|^{2}+2 \delta \sum_{t=1}^{T}\left(\left|Q x_{1}\right|^{2}+\left|x_{2}(t)\right|^{2}\right)+\sum_{t=1}^{T} \eta\left|Q x_{1}\right|+\sum_{t=1}^{T} \eta\left|x_{2}(t)\right|+C_{6} T \\
& \leqslant-\frac{1}{2} \lambda_{1}\left\|x_{2}\right\|^{2}+2 \delta \mu^{2} T+2 \delta\left\|x_{2}\right\|^{2}+\eta \mu T+\eta \sqrt{T}\left\|x_{2}\right\|+C_{6} T \\
& \leqslant\left(-\frac{1}{2} \lambda_{1}+2 \delta\right)\left\|x_{2}\right\|^{2}+C_{7}\left\|x_{2}\right\|+C_{8}
\end{aligned}
$$

where $C_{7}>0, C_{8}>0$ are constants. Note that $\lambda_{1}>4 \delta$, take $\left\|x_{2}\right\|$ sufficient large such that

$$
\sup _{\pi(x) \in S \times V} f(\pi(x)) \leqslant \gamma<\beta .
$$

By all above, the linking conditions (a) and (b) are satisfied. By Lemma 2.1, the system (1.1) possesses $k+1$ periodic solutions.

Proof of Theorem 1.2. Assume that $\left\{\pi\left(x_{n}\right)\right\}$ is a (P.S.) sequence of $f$, i.e., $I\left(x_{n}\right)$ is bounded, $I^{\prime}\left(x_{n}\right) \rightarrow 0$.

For $\lambda_{1}>4 \delta$, we have that

$$
\limsup _{|u| \rightarrow \infty}|u|^{-2} \sum_{t=1}^{T} G(t, u)<-\frac{2 \delta^{2} T}{\lambda_{1}} .
$$

Same as (3.2), we get

$$
\begin{aligned}
\left|\sum_{t=1}^{T}\left(G(t, \tilde{x}(t))-G\left(t, P x_{1}\right)\right)\right| \leqslant & \delta \mu T\left|P x_{1}\right|+\frac{2 \delta^{2} T}{\lambda_{1}}\left|P x_{1}\right|^{2}+\frac{\lambda_{1}}{4}\left\|x_{2}\right\|^{2}+4 \delta \mu^{2} T \\
& +4 \delta\left\|x_{2}\right\|^{2}+\eta \mu T+\eta \sqrt{T}\left\|x_{2}\right\| .
\end{aligned}
$$

For $x \in H_{T}$, we set $\psi(x)=-I(x)$. It is clear that $\psi(x)$ is a G-invariant functional, that is $\psi(x+g)=\psi(x)$, for all $g \in \mathrm{F}, \mathrm{x} \in \mathrm{H}_{\mathrm{T}}$. For all $x \in \mathrm{H}_{\mathrm{T}}$, by (3.11), one has that

$$
\psi(x)=\psi(\tilde{x})=\frac{1}{2} \sum_{t=1}^{T}\left|\Delta x_{2}(t)\right|^{2}-\sum_{t=1}^{T} G(t, \tilde{x}(t))
$$




$$
\begin{aligned}
= & \frac{1}{2} \sum_{t=1}^{T}\left|\Delta x_{2}(t)\right|^{2}-\sum_{t=1}^{T}\left[G(t, \tilde{x}(t))-G\left(t, P x_{1}\right)\right]-\sum_{t=1}^{T} G\left(t, P x_{1}\right) \\
\geqslant & \frac{1}{2} \lambda_{1}\left\|x_{2}\right\|^{2}-\delta \mu T\left|P x_{1}\right|-\frac{2 \delta^{2} T}{\lambda_{1}}\left|P x_{1}\right|^{2}-\frac{\lambda_{1}}{4}\left\|x_{2}\right\|^{2} \\
& -4 \delta \mu^{2} T-4 \delta\left\|x_{2}\right\|^{2}-\eta \mu T-\eta \sqrt{T}\left\|x_{2}\right\|-\sum_{t=1}^{T} G\left(t, P x_{1}\right) \\
= & \left(\frac{1}{4} \lambda_{1}-4 \delta\right)\left\|x_{2}\right\|^{2}-\eta \sqrt{T}\left\|x_{2}\right\|-\delta \mu T\left|P x_{1}\right| \\
& -4 \delta \mu^{2} T-\eta \mu T-\left|P x_{1}\right|^{2}\left(\left|P x_{1}\right|^{-2} \sum_{t=1}^{T} G\left(t, P x_{1}\right)+\frac{2 \delta^{2} T}{\lambda_{1}}\right) .
\end{aligned}
$$

So, $\psi$ is bounded from below.

For $\mathrm{I}\left(x_{n}\right)$ is bounded, $\mathrm{I}^{\prime}\left(x_{n}\right) \rightarrow 0$, there exists $C_{9}$ satisfies $\psi\left(x_{n}\right) \leqslant C_{9}$. By (3.12), we have that

$$
\begin{aligned}
\mathrm{C}_{9} \geqslant \psi\left(\mathrm{x}_{\mathrm{n}}\right)=\psi\left(\tilde{x}_{\mathrm{n}}\right) \geqslant & \left(\frac{1}{4} \lambda_{1}-4 \delta\right)\left\|\mathrm{x}_{2 \mathrm{n}}\right\|^{2}-\eta \sqrt{\mathrm{T}}\left\|\mathrm{x}_{2 \mathrm{n}}\right\| \\
& -\left|\mathrm{P} x_{1 \mathrm{n}}\right|^{2}\left(\left|\mathrm{P} x_{1 \mathrm{n}}\right|^{-2} \sum_{\mathrm{t}=1}^{\mathrm{T}} \mathrm{G}\left(\mathrm{t}, \mathrm{P} x_{1 \mathrm{n}}\right)+\frac{2 \delta^{2} \mathrm{~T}}{\lambda_{1}}\right)-\mathrm{C}_{10}\left|\mathrm{P} x_{1 n}\right|-\mathrm{C}_{11},
\end{aligned}
$$

where $\mathrm{C}_{10}, \mathrm{C}_{11}$ are some positive constants.

Combing $\left(\mathrm{H}_{4}\right)$ with (3.13), we conclude that $\left|\mathrm{P} x_{1 n}\right|$ and $\left\|x_{2 n}\right\|$ are bounded, so $\left\{\tilde{x}_{n}\right\}$ is also bounded. Since $H_{T}$ is finite-dimensional and $\left\{\tilde{x}_{n}\right\} \in H_{T}$, so $\left\{\tilde{x}_{n}\right\}$ contains a convergent subsequence. By $\pi\left(x_{n}\right)=$ $\pi\left(\tilde{x}_{n}\right)$, then $\pi\left(x_{n}\right)$ has a convergent subsequence, that is the functional $\psi$ satisfies the (P.S.) condition.

Hence, all assumptions of Lemma 2.2 are held. Then, by Lemma 2.2 we have that the system (1.1) possesses $\mathrm{k}+1$ geometrically distinct periodic solutions in $\mathrm{H}_{\mathrm{T}}$.

\section{Conclusion}

From the main conclusion, that is, Theorem 1.1 and Theorem 1.2, our results complete and extend some results that of in $[11,16]$. In the last, we would like to point out that based on the results reported in $[1,7,12,13]$ on fractional calculus and time scales we will study some interesting problems, for example, the fractional Hamiltonian system on time scales.

\section{Acknowledgment}

Research was supported by NSFC(11561043). The authors express their sincere thanks to the reviewers and editor for the useful suggestions to improve the paper.

\section{References}

[1] D. Baleanu, F. Jarad, Discrete variational principles for higher-order Lagrangians, Nuovo Cimento Soc. Ital. Fis. B, 120 (2005), 931-938. 4

[2] H.-H. Bin, Subharmonics with minimal periods for convex discrete Hamiltonian systems, Abstr. Appl. Anal., 2013 (2013), 9 pages. 1

[3] C.-F. Che, X.-P. Xue, Infinitely many periodic solutions for discrete second order Hamiltonian systems with oscillating potential, Adv. Difference Equ., 2012 (2012), 9 pages.

[4] H. Gu, T.-Q. An, Existence of periodic solutions for a class of second-order discrete Hamiltonian systems, J. Difference Equ. Appl., 21 (2015), 197-208.

[5] W. Guan, K. Yang, Existence of periodic solutions for a class of second order discrete Hamiltonian systems, Adv. Difference Equ., 2016 (2016), 17 pages. 
[6] Z.-M. Guo, J.-S. Yu, The existence of periodic and subharmonic solutions of subquadratic second order difference equations, J. London Math. Soc., 68 (2003), 419-430. 1, 1, 1

[7] F. Jarad, D. Baleanu, Discrete variational principles for Lagrangians linear in velocities, Rep. Math. Phys., 59 (2007), 33-43. 4

[8] J. Q. Liu, A generalized saddle point theorem, J. Differential Equations, 82 (1989), 372-385. 1, 2.1

[9] Y.-H. Long, Applications of Clark duality to periodic solutions with minimal period for discrete Hamiltonian systems, [Applications of Clarke duality to periodic solutions with minimal period for discrete Hamiltonian systems], J. Math. Anal. Appl., 342 (2008), 726-741. 1

[10] J. Mawhin, M. Willem, Critical point theory and Hamiltonian systems, Applied Mathematical Sciences, SpringerVerlag, New York, (1989). 2.2

[11] X.-H. Tang, X.-Y. Zhang, Periodic solutions for second-order discrete Hamiltonian systems, J. Difference Equ. Appl., 17 (2011), 1413-1430. 1, 1, 1, 4

[12] G.-C. Wu, D. Baleanu, Chaos synchronization of the discrete frational logistic map, Signal Process., 102 (2014), 96-99. 4

[13] G.-C. Wu, D. Baleanu, Z.-G. Zeng, S.-D. Zeng, Lattice fractional diffusion equation in terms of a Riesz-Caputo difference, Phys. A, 438 (2015), 335-339. 4

[14] Y.-F. Xue, C.-L. Tang, Existence of a periodic solution for subquadratic second-order discrete Hamiltonian system, Nonlinear Anal., 67 (2007), 2072-2080. 1, 1, 1

[15] Y.-F. Xue, C.-L. Tang, Multiple periodic solutions for superquadratic second-order discrete Hamiltonian systems, Appl. Math. Comput., 196 (2008), 494-500. 1, 1, 2, 2.4

[16] S.-H. Yan, X.-P. Wu, C.-L. Tang, Multiple periodic solutions for second-order discrete Hamiltonian systems, Appl. Math. Comput., 234 (2014), 142-149. 1, 1, 4

[17] Y.-W. Ye, C.-L. Tang, Periodic solutions for second-order discrete Hamiltonian system with a change of sign in potential, Appl. Math. Comput., 219 (2013), 6548-6555.

[18] Q.-Q. Zhang, Homoclinic orbits for a class of discrete periodic Hamiltonian systems, Proc. Amer. Math. Soc., 143 (2015), 3155-3163. 1 